\title{
Enseñar ciencias: una perspectiva innovadora. Un esquema aproximativo*
}

Dr. Rafael Lucio Gil

Instituto de Investigación de la UCA “Xabier Gorostiaga, S.J.” - IDEUCA

\section{Introducción}

Enseñar ciencias, en un siglo de complejidades y perplejidades, encierra cada día más desafíos y compromisos. Quizás el más importante sea el de acercarnos a las ideas alternativas que tienen profesores y estudiantes sobre las ciencias, como condición para disponernos a enseñar. Cuando a Ausubel, el gran psicólogo del aprendizaje por descubrimiento, le preguntaron los profesores cómo debían enseñar, él respondió: "Primero conozcan las ideas de sus estudiantes y después enséñenles en consecuencia”.

Otro de los ejes sobre los cuales pivota todo el quehacer de los profesores universitarios es la concepción de ciencia que poseen. Por esta razón, mis primeros puntos a abordar serán estos dos precisamente: qué significan estas ideas alternativas y cómo han evolucionado las perspectivas de la Epistemología sobre la Naturaleza de la Ciencia, lo que ofrece grandes aportes a los modelos didácticos más actuales. Estos dos puntos son, de alguna manera, los pivotes sobre los que descansa la posibilidad de que los profesores de ciencias logren realizar cambios en la manera de enseñar ciencias.

En segundo término, comprendidos los dos anteriores que fundamentan un cambiodeperspectiva en la enseñanza, abordaré qué implica enseñar ciencias como algo más que enseñar conceptos y teorías, y la importancia que en este proceso tienen las estrategias superiores de aprendizaje para superar los obstáculos epistemológicos, didácticos y antropológicos en su aprendizaje.

\section{Desmitificar la ciencia y su enseñanza}

Los avances alcanzados en la didáctica moderna de la enseñanza de las ciencias han permitido deducir al menos cinco mitos más generalizados sobre la Ciencia, mitos muy extendidos entre el profesorado de las diversas ciencias y sus estudiantes:

El mito del empirismo beatífico: Desde este mito, profesores y estudiantes consideran que basta con realizar la observación y la experimentación, con el análisis consecuente, para realizar un trabajo científico.

* Conferencia magistral dictada en el IV Congreso Interdisciplinario de Investigación de la Universidad Centroamericana el día 20 de mayo de 2010 en el Auditorio Roberto Terán. 
El mito del idealismo ciego: Desde esta ceguera muy extendida, hay quienes piensan que el conocimiento científico es absoluto, incuestionable, perfecto e independiente de las condiciones sociales, políticas e ideológicas en las cuales se ha desarrollado.

El mito del racionalismo abusivo: Otros piensan que la ciencia nos acerca gradualmente a la verdad sin sufrir cambios bruscos, dependiendo fundamentalmente de la actividad racional e independientemente de las condiciones sociales.

El mito del realismo "naif”: Desde esta posición ingenua basta describir la realidad, lo que ocurre, explicar cómo son los fenómenos y cómo ocurren.

El mito de la verificación crédula: Desde esta perspectiva basta la experimentación científica para conducirnos a la verdad.

Nuestra enseñanza de ciencias habitual, posiblemente, se sienta invadida por alguno o varios de estos mitos o por una mezcla de ellos. En tanto éstos no sean cuestionados, más difícil será adentrarnos en el terreno de una enseñanza auténtica, más humana y vinculada a las limitaciones que tiene la comunidad científica y sus integrantes, y por tanto a las limitaciones y perentoriedad que tienen los modelos científicos que conocemos y enseñamos.

2.1. Las visiones ingenuas del profesorado: La investigación didáctica en todo el mundo ha puesto de manifiesto en los últimos veinte años estos mitos y algunas de sus derivaciones que han penetrado en la enseñanza de los profesores de ciencias:

- La influencia de los medios de comunicación: Éstos se constituyen en perfectos difusores de estereotipos, creencias o visiones inadecuadas de la ciencia y el conocimiento científico. Es por ello que uno de los propósitos que tiene la Academia de Ciencias de Nicaragua, es capacitar a los periodistas para que sepan interpretar y comunicar el conocimiento científico de forma pertinente.

- Las creencias más extendidas demuestran que el modelo más extendido es el Empírico basado en la mera observación y experimentación, con una creencia dogmática en el método científico, método que hoy se sabe tiene múltiples variantes en la comunidad científica y no es el reflejo mecánico de los pasos que los libros de texto nos han enseñado. La idea de máxima objetividad, de verdad absoluta e incuestionabilidad, así como de un conocimiento inmutable, terminal, está ampliamente extendida entre profesores y estudiantes. El filósofo Mikulinski afirmaba que "hoy se enseña la ciencia como se enseñaban los dogmas de la teología en la Edad Media".

- Una comprobación que se ha logrado realizar en estos estudios internacionales es la correlación positiva que existe entre las visiones y mitos que tienen los profesores en distintos contextos de los países, y su abierta relación con la manera como enseñan, los métodos que se utilizan y la relación pedagógica que tienen con sus estudiantes.

- La práctica que generalmente se sigue desde una visión tradicional no es otra que: observar y experimentar para comprobar la verdad. Esta visión limitada explica 
cómo en los años 60' respondió Estados Unidos con el lanzamiento del Sputnik de la Unión Soviética, cuestionándose la calidad de la enseñanza de las ciencias y formulando programas de enseñanza basados en la observación y experimentación que no tuvieron ninguna incidencia en mejorar la enseñanza.

\subsection{Las visiones de los estudiantes, un reflejo de las del profesorado:}

También la investigación didáctica ha penetrado desde hace varias décadas en un fenómeno que anteriormente no era de interés: las concepciones de los estudiantes. En el ámbito de las ciencias, este estudio ha tenido un carácter profundo e incisivo que también ha permeado a otros ámbitos del saber. Entre sus principales conclusiones podemos señalar las siguientes:

- Estas concepciones están profundamente vinculadas a los errores conceptuales que recibieron en la enseñanza previa, y en el contexto cotidiano en el que viven y que incide con gran fuerza en sus visiones de al ciencia.

- Son evidentes los estereotipos que han adoptado sobre los científicos, como grandes sabios, de una capacidad insuperable, con actitudes raras, alejados de la sociedad y con un trabajo científico a prueba de errores.

- Los libros de texto han sido y siguen siendo uno de los principales alicientes que inyectan a los estudiantes y profesores la idea de una ciencia a prueba de todo, dogmática, incuestionable, en la que nunca aparece el error en la actividad científica, y un mundo en el que la mujer ha estado y sigue estando invisibilizada.

- Otra constatación relevante es que estas ideas alternativas tienen un carácter de generalidad en los distintos contextos en que se investigan, son profundamente persistentes, significativas y funcionales, adoptan una estructuración ingenua ("ciencia de los niños"), son fuertemente resistentes al cambio (un reto para la enseñanza), y guardan profundas relaciones con el contexto en el que viven.

\section{Perspectivas sobre la naturaleza de la ciencia}

\subsection{Evolución de la visión de ciencia desde la Filosofía de la Ciencia}

La propia Filosofía de la Ciencia ha venido sufriendo profundos cambios en la medida en que, apoyada en la Sociología y la Psicología de la Ciencia, ha aprendido a comprender mejor la ciencia en sí, el conocimiento científico y las maneras como los científicos se aproximan a él. Tres perspectivas se perciben hoy, por lo general, en las maneras de interpretar la naturaleza de la ciencia. La última es la más actualizada y de mayor consenso: a) Perspectiva Tradicional también denominada Mediacional, b) Nueva Filosofía de la Ciencia, c) El Giro Cognitivo de la Nueva Filosofía de la Ciencia.

a) Perspectiva Tradicional de entender la ciencia y el conocimiento científico: Varios enfoques han madurado en la historia empapados todos ellos de una misma perspectiva. El enfoque de Observación y Experimentación, aún hoy, es el más extendido entre el profesorado y el alumnado. 
Desde 1626 con Francis Bacon -que muriera en la cárcel por oponerse a la visión abstracta que perduró toda la Edad Media- se emprende un camino sin retorno en la observación y experimentación científica con objetividad, de las cuales se inducen o deducen generalizaciones.

En 1650 el Racionalismo de Descartes influenció el saber científico con una perspectiva radicalmente opuesta. Desde él, el pensamiento es el motor del conocimiento científico, lo que hizo que elaborara una racionalidad de la física abstracta que pronto resultó totalmente superada.

En 1642 Galileo Galilei retoma el legado de Bacon, definiendo la metodología científica a partir de la formulación de hipótesis a ser contrastadas por el experimento, a partir de teorías previas y de la combinación de variables. Su planteamiento del Método Experimental con observaciones objetivas del objeto de estudio y con experimentos reales o mentales (Einstein), produjo un gran esplendor en el desarrollo de las ciencias, principalmente la Física.

La corriente positivista, originada con el Círculo de Viena, impuso las reglas de la Lógica a la teoría científica, forzando afirmaciones teóricas desprendidas de afirmaciones observacionales y del método científico debidamente aplicado, garantizando a toda prueba conclusiones incuestionables.

En síntesis, desde esta perspectiva tradicional oscilante entre empirismo, racionalismos y positivismo, el conocimiento científico era considerado comoverdadero e incuestionable, capaz de resolver los grandes problemas de la humanidad. Esta perspectiva bastante generalizada, aunque de manera ingenua, persiste y se niega a evolucionar en la sociedad, las instituciones y la educación en particular.

\subsection{La Nueva Filosofía de la Ciencia: una perspectiva más realista}

Desde los distintos aportes que brindan diversos filósofos de la ciencia a esta perspectiva predomina la idea de que el investigador y la comunidad científica condicionan el conocimiento científico.

Popper, Lakatos y Laudan defienden una racionalidad, pero con matices entre ellos, como el motor del desarrollo de la ciencia.

Para T. Kuhn, con su famoso libro en el que escudriña la evolución de los conceptos y paradigmas de las ciencias, los cambios sociales condicionan los cambios en el conocimiento científico.

Desde otra perspectiva más anárquica, P. Feyerabend cuestiona los métodos de la ciencia, definiendo la falta de una racionalidad y de un método en la ciencia.

EI Falsacionismo de Popper le induce a afirmar que "mil experimentos no confirman una teoría, pero uno sólo la hace falsa". 
En definitiva, el aporte de este modelo de interpretación de la ciencia o Modelo Constructivista de la Ciencia, ha dado un gran aporte a la visión de la ciencia como construcción humana, falible, perfectible, en constante cuestionamiento y avance.

\subsection{La Nueva Filosofía de la Ciencia del Giro Cognitivo}

El modelo conlleva a preservar la racionalidad de la ciencia, para lo cual explica, desde los aportes que brindan las ciencias cognitivas, cómo los científicos utilizan sus capacidades cognitivas interactuando con el mundo para construir el conocimiento científico.

Desde esta óptica, las teorías evolucionan tal como lo hacen las especies, de tal manera que las teorías más sólidas y fuertes prevalecen, en dependencia también de factores sociales, económicos e ideológicos.

Giere, uno de sus máximos exponentes, considera que en el núcleo de toda teoría habita un conjunto de modelos y no de axiomas y leyes. Las hipótesis establecen conexiones entre los modelos abstractos y los modelos reales. Los modelos evolucionan y no son otra cosa que "una forma de mirar la realidad". En síntesis, la racionalidad que defiende es de un Relativismo Moderado, en tanto la ciencia no es independiente sino que depende, también, de los estilos cognitivos del científico y de las fuerzas sociales, económicas e ideológicas.

\subsection{Las dimensiones que caracterizan a la ciencia}

a) Su objetivo: intentar interpretar el mundo.

b) Sus métodos: no tiene un método único y sus resultados son interpretados a la luz de concepciones, resaltándose la unidad dialéctica entre teoría y práctica.

c) La racionalidad: es hipotética en tanto interactúa el objetivo con el contexto a través de hipótesis, lo que hace que sea imposible saber si las teorías son totalmente verdaderas.

d) El Realismo: por cuanto la mediación teórica es con relación a la realidad, al mundo pragmático.

\section{Enseñar ciencias: algo más que enseñar conceptos y teorías}

Los libros de texto que se utilizan y los currículos a los que responden presentan, por lo general, contenidos sin atender a la dinámica que la ciencia sigue en su desarrollo.

En la práctica, además de reforzar las ideas alternativas que traen consigo los estudiantes, se inducen otras nuevas. Se refuerza la idea de la objetividad absoluta -las ideas son siempre verdaderas sin importar cómo es que se han generado y evolucionado- y a lo que se da importancia es sólo al estado final de las teorías, no a su génesis ni procesos de construcción.

Estas ideas transmitidas por el currículum, los programas, los textos y el profesorado, se amalgaman con las que ya poseen los estudiantes, provocándose fuerte resistencia a cambiar concepciones, en definitiva a aprender, lo que plantea un reto muy serio a la didáctica de la enseñanza, al profesorado. 
Cuando en el aula se omiten estos procesos y visiones referidos a la naturaleza de la ciencia, se induce a los alumnos a que oculten sus ideas alternativas, en tanto creen que serán penalizadas en la evaluación, escogiendo la vía más fácil de reproducir, mecanizar sin significado, sin cuestionamiento ni reflexión crítica alguna. En conclusión, aprenden a vivir una perspectiva esquizofrénica: por una parte, estudian, almacenan saberes para el examen, pero continúan sin cambiar sus concepciones en la vida real.

Si sólo tiene valor la teoría final, los alumnos se refugian en el "silencio" (clases silenciosas), sin debate alguno, donde no merece la pena discutir frente a una teoría final apabullante. Se obvia, así, que la comunidad científica llega a construir el saber científico a partir del debate y discusión permanente de ideas. Esta realidad también conduce a suprimir cualquier escapatoria a la creatividad, al pensamiento crítico alternativo, a pensar diferente. Frente a un pensamiento absoluto, terminal y dogmático, resulta imposible un ambiente propicio al pensamiento divergente, creativo, crítico y propositivo.

\subsection{Algunas consecuencias de lo anterior:}

- En general, en los procesos de la ciencia y de su enseñanza no se potencian valores y actitudes de honestidad, racionalidad, autocrítica, perseverancia y objetividad.

- La dependencia que ésta tiene de intereses ideológicos y económicos produce contradicciones entre los "valores proclamados" y los "valores aplicados".

- Un mejor conocimiento de la naturaleza de la ciencia condiciona la selección de contenidos y métodos.

- El proceso científico no debe reducirse al didáctico, pero éste, que es más asequible para el estudiante, no debe contradecir el primero.

Enseñar ciencias supone que los estudiantes se familiaricen con procesos que integran funciones como éstas: observar, comparar, identificar, clasificar, medir, recoger y organizar datos, inferir, predecir, verificar, formular hipótesis, aislar y controlar variables, resolver problemas, comunicar resultados.

\subsection{Un modelo de enseñanza como investigación}

En las últimas décadas se han generado modelos de enseñanza como investigación. Daniel Gil es su principal exponente. Desde esta perspectiva didáctica, muy pertinente para la educación superior, los principales pasos que se deben dar al pretender enseñar un tema con estrategias investigativas, son los siguientes:

a) ¿Qué problemas están en el origen de las teorías relativas al tema?

b) ¿̇Cuáles son o fueron los obstáculos que se debieron superar para lograr avanzar en la solución de los problemas planteados?

c) ¿QQué ideas o razonamientos pueden tener los estudiantes sobre los aspectos anteriores y que pudieran ser obstáculos para el aprendizaje?

d) ¿QQué plan concreto de Investigación y qué secuenciación se puede concertar con los alumnos para que éstos avancen en la solución de los problemas que fueron planteados al inicio? 
Supone, al iniciar cada unidad, plantear situaciones problemáticas que, inspirándose en las que desde el punto de vista histórico o epistemológico, están en el origen de los conocimientos implicados, sirvan de punto de partida para el trabajo de los estudiantes.

A partir de ello, diseñar la secuenciación de los temas del curso con una lógica problematizadora. Organizar el índice de cada uno de los temas/problemas de forma que responda igualmente a una posible estrategia para avanzar en su solución. En este contexto de resolución de problemas, los conceptos y modelos se van introduciendo por parte de los alumnos y del profesor como tentativas, como hipótesis fundadas que deben ser puestas a prueba. Consideramos esencial la realización de recapitulaciones periódicas, que han de ser problematizadoras, sobre lo que se ha avanzado en el problema planteado.

Al preparar la estructura general de un tema podrían seguirse estos pasos:

- Introducción y planteamiento del problema: donde se discute cómo ubicar el tema para que se apropien del problema planteado.

- Índice de la posible estrategia: se discute qué estrategia podría seguirse para avanzar en la solución de problemas de una manera lógica.

- Desarrollo de la estrategia: Se incluye la visión de los conceptos y/o modelos a seguir que actuarán como hipótesis de trabajo. Estos supuestos son puestos a prueba mediante trabajos prácticos, resolución de problemas de "papel y lápiz", prediciendo nuevos hechos.

- Perspectivas abiertas: a nuevos problemas planteados.

\subsection{Posiciones a la hora de enseñar ciencias}

Varias posiciones pueden ser asumidas al enseñar ciencias: desde una Posición Utópica, ubicándose desde un Modelo Crítico, o desde una Perspectiva Paradojal.

- Desde una Posición Utópica: ubicando la ciencia en la perspectiva de aportar a la construcción de una sociedad utópica, de un modelo de desarrollo humano, amigable con el planeta Tierra.

- Desde un Modelo Crítico: cuestionando los modelos tradicionales de hacer ciencia. Es un modelo revolucionario en cuanto a las formas de construir conocimientos y de consumir, y no está consensuado socialmente.

- EI Modelo Paradojal: refleja cuáles son los nuevos objetivos de consumo y cómo aprender a utilizar la tecnología.

\section{Factores que condicionan el problema de enseñar ciencias}

Son muchos los factores que interaccionan y condicionan la enseñanza de cualquier ciencia, entre los que podemos mencionar los siguientes:

- Los cambios sociales que se producen y las consecuentes políticas educativas son un factor determinante que afecta la forma de enseñar ciencias. 
- Los cambios que se están produciendo en la Epistemología de las Ciencias, que modifican las perspectivas didácticas para enfocar la enseñanza de las ciencias.

- El desarrollo de las tecnologías de información y comunicación que posibilitan enormes alcances y recursos para enseñar ciencias.

- EI desarrollo de las Ciencias de la Educación y de la Psicología que ofrecen nuevos descubrimientos y estrategias sobre cómo se aprende y de qué manea lo que se aprenda sea más significativo y duradero.

\subsection{Los cambios en la Epistemología de las Ciencias transforman también su enseñanza}

Cada día son mayores los retos que la ciudadanía tiene, especialmente los niños y jóvenes, para entender la información, comunicar ideas, contrastarlas y ponerlas en crisis.

Los problemas y fenómenos de la vida cotidiana con los que entran en contacto los jóvenes son complejos, interdisciplinarios, razón suficiente para que cada día tengamos que abandonar una enseñanza de las ciencias unidisciplinar. Ello exige que debamos tener nuevos criterios para seleccionar los contenidos de enseñanza desde estas perspectivas.

La vocación de la ciencia es ser explicativa, interpretativa, examinar los fenómenos no desde una visión clásica lineal, sino dinámica, compleja, de enfoque caótico. Los fenómenos de la naturaleza no son lineales, todos ellos se nos presentan desde una perspectiva que sólo la ciencia de la complejidad ha sido capaz de aclarar. El fracaso en las políticas ambientales y de conservación radica, precisamente, en su visión corta de vista, lineal. El reto es avanzar en esta nueva perspectiva, en tanto la enseñanza de las ciencias cada vez más se adentra a desentrañar los fenómenos desde esta nueva perspectiva. Cada vez más la enseñanza de las ciencias está llamada a contribuir a desdibujar las fronteras que históricamente han separado las Ciencias Sociales de las Ciencias Naturales y Experimentales.

\subsection{Las líneas de investigación más actuales en la enseñanza de las ciencias}

Se trata de un tema en constante y dinámica discusión e investigación, centrada principalmente en los aspectos siguientes:

- Valoración del aprendizaje como proceso de largo plazo

- Cambios en la formación del profesorado

- Diversificación de recursos para enseñar ciencias utilizando las TIC's

- Revisión del concepto de evaluación

- Promoción de la autonomía del alumno

- Aprendizaje como construcción social

- Aprendizaje científico: aprender en función de teorías y modelos, y procesos de modelización

- Revisión de objetivos y contenidos desde una ciencia integrada

En todas estas líneas de investigación ya la comunidad científica viene realizando propuestas que aún merecen madurar en el concierto del debate científico. 


\subsection{Algunos factores que influyen en la manera de enseñar ciencias}

Todos ellos son relevantes y actúan como un sistema que se intercomunica generando sinergias que refuerzan o debilitan, según el caso, la calidad de la enseñanza de las ciencias:

- El conocimiento científico y didáctico del profesor-profesora: Nadie enseña lo que no sabe. Si es muy importante saber aplicar métodos, técnicas y estrategias de enseñanza, no lo es menos que éstos apenas son mediadores del conocimiento propiamente dicho. Reforzar un ángulo en demérito del otro afecta negativamente la enseñanza; velar para que ambos ángulos sean del dominio del profesor, es apostar al éxito de la enseñanza de las ciencias.

- Los componentes tecnológicos y técnicos. Éstos están cambiando constantemente y son diversos y variados, por lo que se requiere que el profesorado se mantenga actualizado sobre las potencialidades que ofrecen estos recursos.

- El componente ideológico y axiológico: La ciencia no es neutra. La selección curricular de contenidos tampoco. El profesorado tiene opciones ideológicas y axiológicas. El tema de la ética de la ciencia cada día ha de integrar más como eje transversal la enseñanza de las ciencias.

- Los componentes e ingredientes personales del profesorado: Su personalidad, el arte de enseñar, la capacidad de comunicar, la empatía, en fin, la inteligencia emocional que logre poner en acción en el aula, se convertirá en el principal atractor de los jóvenes hacia el estudio de la ciencia.

\section{Enseñar ciencias aplicando estrategias superiores de aprendizaje}

Si hasta ahora ha sido importante enseñar a aprender ciencias, en adelante lo más importante será enseñar a aprender a aprender ciencias. La investigación didáctica en los últimos años ha comprobado que la utilización de recursos estratégicos superiores de aprendizaje se constituye en el principal dinamizador de capacidades de aprendizaje de los estudiantes.

La Actividad Metacognitiva y la Autorregulación se comportan como el dinamizador estratégico de cambios conceptuales y prácticos fundamentales en la enseñanza y aprendizaje de las ciencias.

Desde la Actividad Metacognitiva el profesorado enseña a sus estudiantes a pensar sobre cómo piensan y cómo actúan en tres dimensiones:

- Metacognición en el conocimiento: El profesorado enseña a aprender cuestionándose, revisándose, deconstruyendo lo mal aprendido, examinando qué es lo que falta por aprender y revisando la calidad de lo que está aprendiendo. 
- Metacognición en la tarea: El profesorado de ciencias enseña a aprender monitoreando, evaluando las tareas que realiza, identificando errores, vacíos y debilidades.

- Metacognición en las estrategias que aplica: El profesorado enseña a los alumnos a ser expertos, aprendiendo a evaluar la efectividad de las estrategias que utilizan para aprender ciencias.

El ejercicio de la actividad metacognitiva conlleva necesariamente y de forma efectiva a la Autorregulación, a la toma de decisiones para superar vacíos, errores, complementar lo que se sabe, cuestionar lo que se cree que se sabe, en fin, modificando constantemente la capacidad de aprender.

Que los estudiantes aprendan a utilizar estos dinamizadores estratégicos potentes, dependerá de que sus profesores también lo hagan y modelicen su práctica en el aula. No lo aprenderán espontáneamente, esto requiere de una orientación guiada que gradualmente delegue al estudiante una autonomía creciente en la utilización de estas estrategias. El uso de estas estrategias superiores hace la diferencia entre una enseñanza moderna y una enseñanza estratégica. No todo lo moderno lleva al máximo desarrollo de capacidades.

Desde la Teoría de la Actividad apuntada por la Escuela Soviética, tres son las partes de la cognición que figuran como capacidades cognitivas y metacognitivas, las cuales, generalmente, son muy poco aplicadas por profesores y alumnos, pero cuya potencialidad hemos podido comprobar en investigaciones didácticas:

- Parte orientadora de la acción:Desde ésta, tanto profesores como estudiantes aprenden a: representar-comprender la acción, anticipar sus resultados, y planificar la acción. Cuando las tres funciones logran articularse exitosamente, la acción de aprender es sumamente exitosa. Si una de ellas falla, afectará a las demás y al resto de funciones. Siendo tan importante, al enseñar ciencias se comprueba que, por lo general, los estudiantes no comprenden, ni representan las acciones que deben realizar para aprender y, por tanto, no las planifican bien ni anticipan los resultados que pretenden. Los estudiantes expertos sí activan estos tres criterios de la acción al aprender.

- Parte reguladora de la acción: Gracias a ella profesores y alumnos revisan lo que enseñan y lo que aprenden respectivamente, la calidad de las tareas que tienen entre manos, las investigaciones que realizan, los problemas que resuelven, la tareas que presentan. Al revisar y monitorear cómo las están resolviendo, detectan limitaciones, errores e insuficiencias y toman decisiones autorregulando para mejorarlas.

- Parte ejecutora de la acción: Es evidente que esta parte es la que nos permite a profesores y estudiantes realizar las acciones tendientes al aprendizaje, pero si no se activan las otras dos, la acción se vuelve mecánica e infructuosa. Gran parte del fracaso del estudiantado en el aprendizaje de las ciencias se debe, precisamente, a que sólo ponen en ejercicio la parte ejecutora, dejando adormecidas las dos partes más importante, la Parte orientadora de la acción y la Parte reguladora de la acción. 
EI común denominador de estas tres funciones, tan pobremente atendidas en la enseñanza habitual de cualquiera de las ciencias, es la Actividad Metacognitiva y la Autorregulación que sirven como telón de fondo activador, cuestionador, que dinamiza los tres resortes descritos. No hacer uso de estos recursos estratégicos, en un siglo tan comprometedor como éste, sería condenar a los estudiantes a aprender para el siglo pasado y al país a desarrollar más aún el subdesarrollo.

\subsection{Enseñar y aprender ciencias son procesos de construcción social}

Si la construcción del conocimiento científico por la comunidad científica invoca un conjunto de escalones insustituibles, la enseñanza de la ciencia no debe distanciarse de ellos, más bien, encuentra su inspiración en este enfoque social socioconstructivo. Estos son sus principales ingredientes que no deberían faltar en las aulas de ciencias:

- Partir de la experiencia, de la percepción: Las experiencias previas con las ideas alternativas que los estudiantes generan en ellas, son un punto de inicio indispensable para que la construcción de conocimiento se logre dar. Discutir estas ideas para generar conflictos sociocognitivos en ellos de manera que las lleguen a cuestionar para aceptar las nuevas, es una tarea que no es fácil y está en las manos del profesor en su desempeño didáctico. Éste ha de ser el punto de partida para avanzar en la construcción del saber.

- Estrategias de razonamiento: Son indispensables para lograr que los estudiantes activen su potencial intrapsicológico poniendo en acción los recursos del razonamiento inductivo y deductivo desde una perspectiva lógica.

- Interacciones socioculturales: El conocimiento en la historia del saber científico se ha construido a partir del debate, la discusión de resultados de investigación y experimentación. Compartir las representaciones sociales y culturales es parte también de la enseñanza y el aprendizaje de las ciencias.

- EI lenguaje: Para Vigotsky el lenguaje forma parte consustancial del desarrollo de las funciones superiores humanas. Gracias al lenguaje, que contribuye a la comunicación interpsicológica, es que el conocimiento que primero es social, pasa a interiorizarse, a ser intrapsicológico. El lenguaje es el mediador fundamental del aprendizaje. Enseñar y aprender sin compartir, sin verbalizar no es enseñar ni aprender.

- Las emociones: Los últimos descubrimiento de la neurolingüística ponen de manifiesto que la unidad de la persona exige que no sólo atendamos los aspectos cognitivos, sino que éstos tienen sentido en tanto las emociones que los albergan tienen cauces adecuados. La neuroeducación, el neuroaprendizaje, la neurociencia, han abierto cauces impensables en la importancia que tiene en el aprendizaje de las ciencias el trabajo con las emociones. La inteligencia emocional, en tal sentido, se convierte en un dinamizador extraordinario para motivar y dinamizar aprendizajes duraderos y relevantes. 


\subsection{Lo existente condiciona la percepción y lo que se aprende en ciencias}

Los descubrimientos logrados por las ciencias cognitivas y, en especial por la psicología cognitiva, nos muestran que lo que aprendemos pasa por un proceso complejo que aún hoy no ha logrado ser descifrado completamente (qué fácil sería enseñar si esto lo supiéramos).

Los alumnos tienen sus propias teorías ingenuas no científicas sobre lo que les queremos enseñar. Por tanto, compiten con los contenidos científicos que presenta el profesorado con las representaciones mentales ingenuas que ya tienen. Pero no sólo es esto, sino que lo que define qué será lo que el alumno asuma de lo que le provee la enseñanza de las ciencias por el profesor o profesora, está determinado por el carácter que tengan estas ideas alternativas. Por tanto, actúan como filtro.

De allí la importancia que tendrá trabajar con tales ideas y teorías (llamadas teorías ingenuas, teorías de los niños, miniteorías, etc.). Pero ¿̇de qué dependerá el éxito de la actividad didáctica? Aquí reside la pregunta central: la planificación didáctica, la visión epistemológica que tenga el profesorado, son determinantes en este proceso para lograr que los alumnos modifiquen sus representaciones ingenuas mejorándolas por la enseñanza.

Tiempo atrás algunos autores hablaron del "cambio conceptual”. Hoy se sabe que exigir este cambio conceptual sería como pedirles a los alumnos renunciar a sus ideas ingenuas par aceptar las nuevas. La complejidad de la cognición nos ha mostrado en los últimos años, que pretender esto sería como exigirles a los alumnos una "revolución copernicana” en sus saberes. Hoy se sabe que lo que ocurre no es eso, sino una evolución gradual, con modelos que evolucionan progresivamente.

\subsection{Algunas fases pertinentes recomendadas para enseñar ciencias con unidades didácticas}

La unidad didáctica representa la agrupación de contenidos que responden en conjunto a una unidad temática con un tratamiento didáctico específico. Algunas de las principales fases que no pueden faltar en una unidad didáctica para que represente un espacio propiciador de aprendizajes, en primera instancia, significa ubicar al estudiante ante un conjunto de actividades de aprendizaje a realizar, contextualizadas, interesantes, investigativas, a través de las cuales logre poner en cuestión sus conocimientos y construir otros nuevos.

Ubicados en un sistema de referencia, el Eje X significa el avance entre la posición más SIMPLE a lo más COMPLEJO. Se inicia por situaciones muy simples, avanzando progresivamente hacia otras más complejas. En el Eje Y avanzamos desde situaciones más CONCRETAS hacia situaciones progresivamente más ABSTRACTAS. La combinación armónica de fases en estas dos dimensiones, hace que los estudiantes logren avanzar desde situaciones SIMPLES Y CONCRETAS, hacia otras progresivamente más ABSTRACTAS pero también más COMPLEJAS. 
Algunas de las principales fases que facilitan procesos de aprendizaje significativo son las siguientes:

Fase de familiarización y exploración: oportunidad inexcusable para conocer las ideas alternativas, las expectativas sobre el tema, de forma que se familiaricen inicialmente con sus aspectos más relevantes.

Fase de introducción de nuevos puntos de vista (el saber científico): ante la discusión de las ideas alternativas y de sus expectativas, se ha preparado el terreno para afianzar su motivación a comprender qué dice la ciencia al respecto, qué conceptos son fundamentales y cómo éstos deben ser puestos a prueba con ejercicios, experimentos, problemas, estudios de caso, etc.

Fase de actividades de síntesis y reestructuración conceptual: en ella los estudiantes logran integrar, articular, relacionar, comprobar tales conceptos, avanzando en su nivel de abstracción. Aquí se dan procesos mentales de reestructuración de conceptos, de cambios significativos en las formas de pensar y ver los fenómenos en estudio.

Fase de aplicación: una vez que se ha avanzado en el nivel de abstracción, es necesario progresar en el nivel de COMPLEJIDAD. Por ello la aplicación se logra en varios estadios. El primero es la aplicación a nivel académico, y el segundo estadio es en el nivel de la realidad, del contexto cotidiano. Es evidente que preparar un proyecto de curso significará la integración de todos los procesos y conocimientos en la puesta en práctica de los mismos en el terreno de la práctica, de la realidad. Cuando las actividades de aplicación quedan rezagadas únicamente a un contexto académico y no real, difícilmente el estudiante sabrá trasladar lo aprendido a estos contextos en los que nunca antes aprendió a aplicar lo aprendido.

\section{En síntesis}

Estas ideas rectoras nos quedan como aprendizaje:

- Una visión epistemológica actualizada del saber científico y su incidencia en la enseñanza de las ciencias

- Comprensión de las finalidades de la ciencia

- Superación de los mitos que tenemos sobre la ciencia

- Comprensión de las ideas alternativas de los estudiantes como puntos de partida para ayudarles a superarlas

- Comprensión de cómo se aprende para enseñar en consecuencia

- Adoptar una visión constructivista de la enseñanza y aprendizaje de las ciencias.

- Enseñar ciencias aplicando estrategias superiores de aprendizaje como dinamizadores efectivos.

- Enseñar ciencias como investigación. 